Artículo de investigación E16A10 * Proyecto "Determinantes del comportamiento sostenible en universidades del noreste de Méjico: un análisis exploratorio”. * Universidad Autónoma de Tamaulipas. * Recibido: 10.11.2019. Aprobado versión final: 26.12.2019. JEL: L2, Q5. * Pp. 131-152. * doi: 10.33571/teuken.v11n16a6

\title{
Determinantes del comportamiento sostenible de las universidades del noreste de Méjico.
}

\author{
Un análisis exploratorio.
}

\section{Determinants of sustainable behavior of universities in northeastern Mexico.}

An exploratory analysis

\section{Lucero de Jesús Rodríguez Jasso - Mónica Lorena Sánchez Limón \\ Arturo Briseño García \\ MÉJICO}

Resumen: La sostenibilidad ha emergido con la finalidad de mejorar la calidad de vida de los seres vivos en el planeta. El objetivo del artículo es determinar los factores que impulsan el comportamiento sostenible de las universidades del noreste de Méjico. Los resultados se logran a partir de una investigación cualitativa de corte exploratorio, donde se establecieron los elementos internos y externos más relevantes de dicho fenómeno. El método utilizado consistió en un estudio de casos en universidades del noreste de Méjico, identificándose como principales determinantes del comportamiento sostenible elementos a nivel individual, institucional y contextual, tales como los marcos normativos, las competencias existentes, la presencia o ausencia de una conciencia, y las actitudes.

Palabras clave: comportamiento sostenible; sostenibilidad; determinantes; universidades mejicanas.

Abstract: Sustainability has emerged in order to improve the quality of life for living things on the planet. The objective of the article is to determine the factors that drive the sustainable behavior of the universities of northeast Mexico. The results are achieved from a qualitative exploratory research, where the most relevant internal and external elements of this phenomenon were established. The method used consisted of a case study at universities in northeast Mexico, identifying as individual determinants of sustainable behavior the main elements at the individual, institutional and contextual levels, such as regulatory frameworks, existing competences, the presence or absence of an awareness, and attitudes.

Keywords: sustainable behavior; sustainability; determinants; mexican universities.

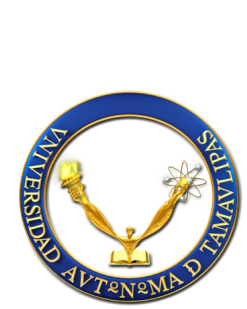

Lucero de Jesús Rodríguez es Máster en Dirección Empresarial con énfasis en Administración Estratégica y Estudiante de Doctorado en Ciencias Administrativas de la Universidad Autónoma de Tamaulipas, Méjico.

Contacto: lucero.rdzj@gmail.com

Mónica Lorena Sánchez Limón es Doctora en Ciencias de la Administración y Profesora de la Universidad Autónoma de Tamaulipas, Méjico.

Contacto: msanchel@docentes.uat.edu.mx

Arturo Briseño García es Doctor en Administración de Empresas y Profesor de la Universidad Autónoma de Tamaulipas, Méjico.

Contacto: abriseno.dca@gmail.com 


\title{
Determinantes do comportamento sustentável em universidades do nordeste do México: uma análise exploratória.
}

\begin{abstract}
Resumo: A sustentabilidade surgiu para melhorar a qualidade de vida dos seres vivos do planeta. O objetivo do artigo é determinar os fatores que impulsionam o comportamento sustentável das universidades do nordeste do México. Os resultados são obtidos a partir de uma pesquisa exploratória qualitativa, onde foram estabelecidos os elementos internos e externos mais relevantes desse fenômeno. O método utilizado consistiu em um estudo de caso em universidades do nordeste do México, identificando como determinantes individuais do comportamento sustentável os principais elementos nos níveis individual, institucional e contextual, como estruturas regulatórias, competências existentes, presença ou ausência de consciência, e atitudes.
\end{abstract}

Palavras-chave: comportamento sustentabel; sustentabilidade; determinantes; universidades mejicanas.

\section{Introducción}

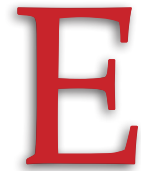

I artículo es el resultado de una investigación de corte exploratorio y descriptivo cuyo enfoque de análisis se dirige hacia un grupo de universidades con prácticas sostenibles ubicadas en las zonas de Tamaulipas, Nuevo León, Coahuila y San Luis Potosí en Méjico. La investigación se desarrolló desde la perspectiva inductiva y cualitativa apoyándose en el análisis de información recopilada a través de entrevistas semi-estructuradas; se basó en un método de análisis de casos múltiples para examinar los determinantes de un comportamiento sostenible.

En las últimas décadas, la población mundial ha atravesado por una serie de acontecimientos que han repercutido en su calidad de vida; por un lado, una explotación excesiva y no sostenible de los recursos naturales; por el otro, un consumismo cada vez más alto. Esto ha dado como resultado un agotamiento del planeta, pero también la necesidad de un cambio en el manejo de los recursos naturales y los hábitos de consumo (Aguiñaga, Heriques, Scheel \& Scheel, 2018:2; IPCC, Pachauri \& Meyer, 2014:8). Por otro lado, la presencia de la marginación y exclusión de grupos vulnerables demanda la atención para actuar al respecto y minimizar los efectos en la sociedad (Martin \& Osberg, 2007:35).

La sostenibilidad es un concepto de reciente estudio, cuya relevancia ha ido creciendo por la presencia de fenómenos ambientales y sociales que han afectado la calidad de vida de las personas. Al tener como enfoque la integración de las dimensiones económico, social y ambiental se genera 
una perspectiva más amplia que permite el cumplimiento de objetivos actuales y de largo plazo (Urbaniec, 2018).

Debido a la constante controversia generada por la utilización indistinta de los conceptos de "sostenibilidad" y "sustentabilidad", para efectos del presente estudio se adoptará la denominación de "sostenibilidad", concepto utilizado por la Organización de las Naciones Unidas (ONU) en la presentación de los Objetivos de Desarrollo Sostenible (ONU, 2018; Urbaniec, 2018).

Por su parte, un comportamiento forma una de las bases principales para el reconocimiento de oportunidades y permite actuar frente a ellas (Cope, 2005:377; Hwee Nga \& Shamuganathan, 2010:261). Es un conjunto de predisposiciones personales que guían las acciones y la autoexpresión (Lülfs \& Hahn, 2014:50; Vuorio, Puumalainen \& Fellnhofer, 2017:6). El comportamiento sostenible es un conjunto de acciones dirigidas hacia la preservación del entorno natural (incluyendo el aspecto cultural) y del entorno social sin descuidar la parte del desarrollo económico (Bansal \& Roth, 2000:687; Lülfs \& Hahn, 2014:44).

Las acciones y decisiones desprendidas de un comportamiento establecen el desempeño general de una institución (Lülfs \& Hahn, 2014:43), por lo que el conocimiento de los factores que influyen en el proceso cognitivo resultan de gran relevancia para la explicación del fenómeno de estudio. Algunos autores han orientado sus investigaciones a la explicación del fenómeno del comportamiento sostenible, involucrando elementos relacionados, principalmente, con el marco normativo, la intención, las competencias y la actitud, entre otros (Gast, Gundolf \& Cesinger, 2017; Lülfs \& Hahn, 2014; Muñoz, 2017; Roysen, 2018; Tur-Porcar, Roig-Tierno \& Llorca Mestre, 2018).

En la investigación se exploraron los factores internos y externos de la unidad de estudio como elementos de influencia en la generación de un comportamiento sostenible y de algunos factores a nivel individual, institucional y contextual. Las variables relacionadas para la elaboración de una codificación previa se identificaron con base en los resultados de estudios anteriores, que mostraban la importancia de las intenciones, normas sociales, actitudes, valores personales y competencias.

El artículo tiene como objetivo identificar los determinantes que impulsan el comportamiento sostenible en las universidades del noreste de Méjico, con el fin de validar lo propuesto por los autores previos, brindando de manera empírica un panorama más integrador de los factores que 
intervienen en el fenómeno. El artículo se estructura a partir del marco teórico sobre el problema, seguido de la fundamentación metodológica, el análisis de resultados y las conclusiones.

\section{Revisión de literatura}

La búsqueda de información se llevó a cabo a través de buscadores de investigación científica, como Scopus, Emerald, Elsevier, Science Direct, y WebofScience. Las palabras clave utilizadas para llevar a cabo la búsqueda fueron: "sostenibilidad", "comportamiento sostenible", "factores sostenibles", "impulsores sostenibles", "intención sostenible", entre otros. A pesar de que alguna literatura "gris" (datos del GEM, INEGI y ONU) fue utilizada para desarrollar algunas conceptualizaciones, gran parte del análisis se basó en artículos científicos.

Como resultado del análisis de la literatura se encontró que la ONU (2018) presenta 17 objetivos que marcan la pauta para un actuar sostenible y sirven como guía para los países, organizaciones y empresas para la generación de prácticas sostenibles. Por otro lado, del estudio de Zdanyté, Neverauskas \& Sabaliauskaité (2014) se infiere que, no solamente la presencia de una filosofía es indispensable en el contexto sostenible, sino que la presencia de ciertas competencias permite llevar a cabo con éxito esta filosofía. Dentro de las competencias se menciona el desarrollo de métodos adecuados para obtener un involucramiento de todos los actores y una correcta implementación de las directrices.

Otros autores como Muñoz (2017) y Roysen (2018) resaltan la importancia de las competencias en el actuar sostenible, al igual que Lloyd \& Evans (2016); Criado-Gomis, Cervera-Taulet \& Iniesta-Bonillo (2017); DiVito \& Bohnsack (2017); Lans, Blok \& Wesselink (2014) y Ploum, Blok, Lans \& Omta (2018), quienes identifican una serie de competencias necesarias para este efecto, tales como: eficiencia, innovación, tener un sistema de pensamiento, prospección, diversidad e interdisciplinariedad, acción, gestión, entre otros.

Algunas investigaciones (Koe, Omar \& Majid, 2014; Koe, Omar \& Sa'ari, 2015; Lülfs \& Hahn, 2014; Muñoz, 2017; Vuorio et al., 2017) concuerdan con la teoría del comportamiento planeado con relación a la importancia que ejercen las intenciones sobre un comportamiento sostenible, en donde estas son afectadas por las actitudes. Adicionalmente, autores como Criado-Gomis et al. (2017); Gast et al. (2017); Imtiaz, Blundel \& Schaefer (2017); Jahanshahi, Breem \& Bhattacharjee (2017); Muñoz (2017); Roysen 
(2018) señalan la importancia de los valores sostenibles, las normas y la presión social en el presente fenómeno de estudio. Dentro de los valores mencionados se encuentran: el altruismo, la empatía, la ética, el valor prosocial, el ecocentrismo y la recompensa intrínseca (Ruiz-Ruano \& Puga, 2016; Tur-Porcar et al., 2018; Vuorio et al., 2017).

A partir de lo anterior, se identifica que las intenciones, las actitudes, el marco normativo y las competencias se pueden tomar como variables relacionadas preliminares para el presente estudio. A continuación, se realiza una descripción de cada una de ellas.

\section{Teoría del comportamiento planeado}

La teoría del comportamiento planeado propuesta por Ajzen (1991:181) establece que la intención y el control del comportamiento percibido (habilidades) son factores que interactúan simultáneamente para lograr el desarrollo de un comportamiento. Las intenciones influyen en el comportamiento en el mismo grado de la presencia de las habilidades, por su parte, las habilidades influyen mientras se tenga una intención hacia el comportamiento.

\section{El comportamiento sostenible y su relación con la teoría del comportamiento planeado}

El comportamiento sostenible son las acciones o prácticas orientadas a la conservación ambiental y cultural, y a una sociedad con una óptima calidad de vida, sin dejar de lado la prosperidad económica y teniendo una visión presente y a largo plazo (Bansal \& Roth, 2000: 687; Jahanshahi et al., 2017: 3; Lülfs \& Hahn, 2014: 44). Es decir, un comportamiento sostenible persigue un equilibrio ambiental, social y económico a través de un conjunto de prácticas.

Para el desarrollo de un comportamiento, la influencia de las intenciones y el control del comportamiento percibido, determinado a través del nivel de habilidades, son elementos de gran relevancia que permiten determinar de una manera más precisa el comportamiento (Ajzen, 1991: 181; Imtiaz et al., 2017: 2; Lülfs \& Hahn, 2014: 54; Vuorio et al., 2017: 4). La presencia de ciertos valores permite la generación de una conciencia que también facilita el proceso (Lülfs \& Hahn, 2014: 54; Torres-Hernández, Barreto \& Rincón, 2015: 88). Lo descrito anteriormente presenta una fuerte vinculación con las bases cognitivas del comportamiento que explica la teoría del comportamiento planeado (Ajzen, 1991). 


\section{La intención sostenible y su relación con el comportamiento sostenible}

Una intención es el paso previo a la generación de un comportamiento y se describe como la fuerza y enfoque que un individuo ejerce para que este comportamiento se lleve a cabo (Ajzen, 1991: 181, 2002: 665; Bird, 1988: 443; Muñoz, 2017: 5; Torres-Hernández et al., 2015: 87). También es aquella donde los esfuerzos enfocados se dirigen a la consecución de los parámetros de la sostenibilidad (Sung \& Park, 2018: 3; Wagner \& Maximilians, 2012: 520) y presenta una influencia directa por parte de las actitudes y normas sociales dentro del mismo contexto (Gast et al., 2017: 48; Lülfs \& Hahn, 2014: 47; Vuorio et al., 2017: 4).

\section{La actitud sostenible y la norma social en su relación con la intención sostenible}

Una actitud se presenta como el estado cognitivo y subjetivo de un individuo que genera la interpretación del entorno que lo rodea, lo que le permite guiar sus acciones respecto a las situaciones que se le presentan (Muñoz, 2017: 5; Torres-Hernández et al., 2015: 87). Una actitud ejerce influencia sobre el comportamiento al impactar en la intención del individuo para llevar a cabo una acción, incidiendo en la percepción que se haya generado previamente (Ajzen, 1991: 188; Koe et al., 2015: 571; Vuorio et al., 2017: 4). Es así que, si el individuo presenta una actitud favorable hacia la sostenibilidad, la probabilidad de generar un comportamiento en el mismo sentido será mayor (Muñoz, 2017: 5; Torres-Hernández et al., 2015: 87).

Las normas sociales se representan a través de las presiones que el individuo percibe por parte de las expectativas de algunas personas de influencia en su entorno, las cuales pueden verse reflejados en los miembros de su familia, los compañeros y jefes de trabajo, ciertas organizaciones de referencia, entre otros, por lo que sus opiniones pueden influir en la intención para realizar una acción (Ajzen, 1991: 188; Koe et al., 2015: 572; Torres-Hernández et al., 2015: 87), lo mismo sucede en el ámbito sostenible (Lülfs \& Hahn, 2014: 47).

\section{Valores sostenibles y su relación con el comportamiento sostenible}

Los valores son criterios que el individuo utiliza para realizar una evaluación sobre una decisión; así mismo. Constituyen la base para la definición de un comportamiento (Hwee Nga \& Shamuganathan, 2010: 259; Jahanshahi et al., 2017: 3; Vuorio et al., 2017: 6). Los valores se pueden adoptar a través del tiempo, las experiencias del individuo y pueden 
generarse a partir de un efecto mimético con las personas que lo rodean (Hwee Nga \& Shamuganathan, 2010: 263; Jahanshahi et al., 2017: 3) al compartir su cultura y sus valores (Tur-Porcar et al., 2018: 3).

Las personas con una orientación sostenible presentan una inclinación paralela de valores dirigidos al cuidado y preservación del medio ambiente, el respeto y ayuda al prójimo y generación de un bienestar económico (Jahanshahi et al., 2017: 4), por lo que las actividades realizadas se dirigen principalmente a la búsqueda de un equilibrio en este sentido (Imtiaz et al., 2017: 4; Muñoz, 2017: 4; St-Jean y Labelle, 2018: 4).

\section{Competencias sostenibles y su relación con el comportamiento sostenible}

Las competencias son el conjunto de habilidades percibidas que el individuo cree tener respecto a la realización exitosa de una actividad (Ploum et al., 2018: 115; Vuorio et al., 2017: 7) y pueden ser explicadas como el nivel de autoeficacia percibida (Vuorio et al., 2017: 7). Las competencias o habilidades percibidas permiten reconocer el nivel de resolución que se puede tener en alguna situación (Ploum et al., 2018: 115; Vuorio et al., 2017: 7), lo que influye en la toma de decisiones (Muñoz, 2017: 5).

Derivado del enfoque cualitativo de la investigación para abordar el fenómeno del comportamiento sostenible, se hace uso de un método inductivo para el establecimiento de proposiciones de investigación, las cuales se desprenden de algunas nociones básicas para identificar elementos no previamente abordados:

PI1: Existen elementos a nivel individual, institucional y contextual que afectan de manera positiva o negativa la generación de comportamientos sostenibles en las universidades del noreste de Méjico.

PI2: Existen elementos del entorno interno y externo de la universidad que afectan de manera positiva o negativa la generación de comportamientos sostenibles en las universidades del noreste de Méjico.

\section{Metodología}

Dada la reciente importancia del tema y derivado de la escasez de resultados empíricos, se establece la necesidad de realizar un estudio con enfoque cualitativo y de tipo exploratorio de corte transversal y no experimental (Tur-Porcar et al., 2018). Se realizó un análisis de casos múltiples exploratorio para conocer las condiciones contextuales 
del fenómeno y las causas por las cuales se genera o se limita el comportamiento sostenible de las universidades del noreste de Méjico (Baxter y Jack, 2008: 545; Westgren y Zering, 1998: 416; Yin, 2003: 5). Dado el número limitado de casos que pueden ser estudiados, es de gran importancia la selección de los mismos de manera crítica, extrema y relevante, en donde el fenómeno pueda ser observable (Lloiyd \& Evans, 2016: 367).

Para incrementar la diversidad y heterogeneidad de los datos, se tomaron cinco universidades públicas y privadas de los estados de Tamaulipas, Nuevo León, Coahuila y San Luis Potosí de Méjico. La determinación de los casos se llevó a cabo principalmente entre las universidades con una filosofía sostenible o que contaran con una unidad de sostenibilidad. Las universidades seleccionadas presentan diferentes prácticas y estrategias para un desempeño sostenible. La disponibilidad y accesibilidad a las unidades de estudio fue determinante para la selección de casos. El contacto previo con cada una de las universidades fue a través del contacto personal, por vía electrónica o telefónica.

\section{Técnica de recolección de datos}

Para llevar a cabo la recolección de datos se establecieron citas previas para la visita a los diferentes lugares de trabajo y, en otros casos, para la determinación del día y hora para la llamada telefónica. Se realizaron seis entrevistas estructuradas a expertos, en su mayoría, titulares de los departamentos de sostenibilidad, con un amplio conocimiento y experiencia en el tema. Las entrevistas exploraron los determinantes internos y externos a la universidad que influyen o limitan el comportamiento sostenible; previo a la aplicación del instrumento, se realizó una prueba piloto de la guía de entrevista.

Para el desarrollo de la entrevista se trató de evitar que el entrevistador hiciera comentarios que pudieran sesgar la respuesta de los sujetos de estudio, y dar flexibilidad a las preguntas, generar la confianza necesaria para mayor comodidad del entrevistado, sin perder en momento alguno la formalidad. El promedio de duración de las entrevistas fue de 40 minutos aproximadamente, obteniendo una respuesta positiva en el $100 \%$ de los casos de los cuales el 3 fueron de manera presencial, 2 de manera telefónica y 1 vía electrónica. Todas las entrevistas fueron plenamente grabadas y transcritas, y se creó una base de datos para cada entrevista. 
La codificación de los datos se llevó a cabo a través de una segmentación de la entrevista, buscando palabras significativas y apoyándose en el software MAXQDA12. Como ejercicio inicial se llevó a cabo una codificación provisional basada en la revisión de la literatura previa; no obstante, durante el desarrollo de la misma se ejerció una codificación y sub-codificación simultánea, descriptiva y de magnitud (Miles, Huberman \& Saldana, 2013). A través de la codificación se establecieron diversas categorías que permitieron realizar un análisis para determinar los elementos de influencia en el comportamiento sostenible y un ejercicio de comparación con respecto a los resultados de estudios previos para brindar un soporte empírico.

Con la finalidad de incrementar la validez del estudio (Manesh \& Rialp-Criado, 2018), los datos fueron triangulados con la información proporcionada por diversas fuentes de evidencia, tales como la recolección de datos por diversos informantes de las universidades, la observación directa (visita física a las instalaciones de las universidades y unidades de sostenibilidad) y los datos secundarios como la información en línea disponible, reportes realizados por las unidades y websites de las universidades.

\section{Análisis de resultados}

Los resultados arrojaron información clave sobre la descripción general de las unidades de análisis (tabla 1), donde aspectos como el género de los entrevistados, el tipo de universidad y el estado de ubicación permitieron identificar la preocupación por el particular. También se encontró que la región predominante en la atención a los problemas sostenibles es el Estado de Nuevo León; no obstante, son las universidades públicas las que tienen la mayoría en la iniciativa (60\%).

Tabla 1. Descripción general de las unidades

\begin{tabular}{|c|c|c|c|c|c|}
\hline Caso & Estado & $\begin{array}{c}\text { Tipo de } \\
\text { universidad }\end{array}$ & Género & $\begin{array}{c}\mathbf{N}^{\circ} \mathrm{de} \\
\text { entrevistas }\end{array}$ & Tiempo \\
\hline U1 & Tamaulipas & Pública & Mujer & 1 & 1:01:31 \\
\hline U2 & Nuevo León & Privada & $\begin{array}{l}\text { Hombre y } \\
\text { mujer }\end{array}$ & 2 & $1: 02: 25$ \\
\hline U3 & Nuevo León & Privada & Hombre & 1 & $0: 33: 25$ \\
\hline U4 & Coahuila & Pública & Mujer & 1 & $0: 30: 00$ \\
\hline U5 & San Luis Potosí & Pública & Hombre & 1 & $0: 46: 16$ \\
\hline
\end{tabular}

Fuente: Elaboración propia (2019). 
Las codificaciones permitieron establecer una comparación y contraste con conjeturas de algunos autores en investigaciones previas relacionadas con el tema. La primera agrupación de datos se enfocó en cuatro categorías (tabla 2), las cuales giraron en torno a los factores generales relacionados al comportamiento sostenible: (1) factores internos positivos, (2) factores internos negativos, (3) factores externos positivos y (4) factores externos negativos.

Tabla 2. Categorización general

\begin{tabular}{|c|l|l|} 
Código & \multicolumn{2}{|c|}{ Denominación } \\
FI+ & $\begin{array}{l}\text { Factores internos } \\
\text { positivos }\end{array}$ & $\begin{array}{l}\text { Elementos dentro de la universidad que se } \\
\text { considera que impulsan al comportamiento } \\
\text { sostenible. }\end{array}$ \\
\hline FI- & $\begin{array}{l}\text { Factores internos } \\
\text { negativos }\end{array}$ & $\begin{array}{l}\text { Elementos dentro de la universidad que se } \\
\text { considera que limitan al comportamiento } \\
\text { sostenible. }\end{array}$ \\
\hline FE+ & $\begin{array}{l}\text { Factores externos } \\
\text { positivos }\end{array}$ & $\begin{array}{l}\text { Elementos fuera de la universidad que se } \\
\text { considera que impulsan al comportamiento } \\
\text { sostenible. }\end{array}$ \\
\hline FE- & $\begin{array}{l}\text { Factores externos } \\
\text { negativos }\end{array}$ & $\begin{array}{l}\text { Elementos fuera de la universidad que se } \\
\text { considera que limitan al comportamiento } \\
\text { sostenible. }\end{array}$ \\
\hline
\end{tabular}

Fuente: Elaboración propia (2019).

Los factores internos se relacionan con los factores internos de la universidad que inciden o fomentan un comportamiento sostenible. Estos pueden estar presentes en los trabajadores y en la gestión organizacional. Los factores se dividen en positivos o negativos, dependiendo del impulso o limitación que estos brinden. En segunda instancia, se establecen todos aquellos factores del entorno externo o ajeno a la universidad que influyen de manera positiva o negativa hacia el desempeño de dicho comportamiento.

La codificación y sub-codificación preliminar de datos se realizó a través de la recopilación de información documental previa relacionada con el comportamiento sostenible $y$, después de analizar y comparar las opiniones de los entrevistados con esa información, se llevaron a cabo algunas modificaciones y adaptaciones a los códigos preliminares para una mejor explicación. Cada caso fue complementando a los otros, al replicar los hallazgos bajo ciertas circunstancias. Siguiendo la exploración basada en las categorías generales establecidas (factores internos y factores externos), se presentó lo siguiente (tabla 3): 
Tabla 3. Aspectos internos y externos de influencia hacia un comportamiento sostenible

\begin{tabular}{|c|c|c|c|c|c|c|}
\hline Dimens. & Categoría & $\% *$ & Criterio & $\% *$ & $\begin{array}{c}\text { Criterios } \\
\text { ponderados }\end{array}$ & $\% * *$ \\
\hline \multirow{15}{*}{$\begin{array}{c}\text { Factores } \\
\text { internos } \\
\text { positivos } \\
(\mathrm{FI}+)\end{array}$} & Normas (NO) & 100 & Valores & 83.3 & Valores & 19.1 \\
\hline & & & Norma social & 50 & Norma social & 5.2 \\
\hline & & & Norma coercitiva & 33.3 & Norma coercitiva & 6.4 \\
\hline & & & Norma mimética & 16.6 & Norma mimética & 0.6 \\
\hline & Edad (ED) & 16.6 & & & Edad & 2.3 \\
\hline & Hábitos (HA) & 33.3 & & & Hábitos & 2.3 \\
\hline & Actitud (AC) & 33.3 & & & Actitud & 4.6 \\
\hline & Recursos (RE) & 33.3 & & & Recursos & 1.2 \\
\hline & Competencias (CO) & 100 & Acción & 83.3 & Acción & 26 \\
\hline & & & $\begin{array}{l}\text { Gestión } \\
\text { estratégica }\end{array}$ & 83.3 & $\begin{array}{l}\text { Gestión } \\
\text { estratégica }\end{array}$ & 5.8 \\
\hline & & & $\begin{array}{l}\text { Diversificación e } \\
\text { interdisciplina- } \\
\text { riedad }\end{array}$ & 66.6 & $\begin{array}{l}\text { Diversificación e } \\
\text { interdisciplina- } \\
\text { riedad }\end{array}$ & 4.6 \\
\hline & & & $\begin{array}{l}\text { Sistema de } \\
\text { pensamiento }\end{array}$ & 66.6 & $\begin{array}{l}\text { Sistema de } \\
\text { pensamiento }\end{array}$ & 4.6 \\
\hline & & & Normativa & 50 & Normativa & 1.7 \\
\hline & & & $\begin{array}{l}\text { Pensamiento } \\
\text { prospectivo }\end{array}$ & 16.6 & $\begin{array}{l}\text { Pensamiento } \\
\text { prospectivo }\end{array}$ & 0.6 \\
\hline & Conciencia & 100 & & & Conciencia & 15 \\
\hline \multirow{11}{*}{$\begin{array}{c}\text { Factores } \\
\text { internos } \\
\text { negativos } \\
\text { (FI-) }\end{array}$} & $\begin{array}{l}\text { Contexto Interno } \\
\text { inadecuado (Cli) }\end{array}$ & 16.6 & & & $\begin{array}{l}\text { Contexto Interno } \\
\text { inadecuado }\end{array}$ & 1.8 \\
\hline & $\begin{array}{l}\text { Hábitos } \\
\text { arraigados (HAa) }\end{array}$ & 16.6 & & & $\begin{array}{l}\text { Hábitos } \\
\text { arraigados }\end{array}$ & 0.9 \\
\hline & $\begin{array}{l}\text { Normas coercitivas } \\
\text { internas } \\
\text { inadecuadas (NCi) }\end{array}$ & 16.6 & & & $\begin{array}{l}\text { Normas coercitivas } \\
\text { internas } \\
\text { inadecuadas }\end{array}$ & 0.9 \\
\hline & $\begin{array}{l}\text { Recursos } \\
\text { limitados (RE) }\end{array}$ & 83.3 & $\begin{array}{l}\text { Recurso } \\
\text { económico }\end{array}$ & 83.3 & $\begin{array}{l}\text { Recurso } \\
\text { económico }\end{array}$ & 13.4 \\
\hline & & & Recurso humano & 16.6 & Recurso humano & 7.1 \\
\hline & $\begin{array}{l}\text { Falta de } \\
\text { conciencia (CON) }\end{array}$ & 50 & & & $\begin{array}{l}\text { Falta de } \\
\text { conciencia }\end{array}$ & 9.8 \\
\hline & $\begin{array}{l}\text { Actitud negativa } \\
\text { (AC) }\end{array}$ & 50 & Consumista & 16.6 & Consumista & 30.4 \\
\hline & & & Apatía & 16.6 & Apatía & 0.9 \\
\hline & & & Resistencia & 16.6 & Resistencia & 0.9 \\
\hline & $\begin{array}{l}\text { Falta de } \\
\text { competencias } \\
\text { internas (COi) }\end{array}$ & 100 & Falta de acción & 100 & Falta de acción & 21.4 \\
\hline & & & $\begin{array}{l}\text { Falta de gestión } \\
\text { estratégica }\end{array}$ & 33.3 & $\begin{array}{l}\text { Falta de gestión } \\
\text { estratégica }\end{array}$ & 6.3 \\
\hline
\end{tabular}




\begin{tabular}{|c|c|c|c|c|c|c|}
\hline Dimens. & Categoría & $\% *$ & Criterio & $\% *$ & $\begin{array}{c}\text { Criterios } \\
\text { ponderados }\end{array}$ & $\% * *$ \\
\hline \multirow{3}{*}{$\begin{array}{c}\text { Factores } \\
\text { internos } \\
\text { negativos } \\
(\mathrm{FI})\end{array}$} & & & $\begin{array}{l}\text { Falta de } \\
\text { habilidad } \\
\text { interpersonal }\end{array}$ & 33.3 & $\begin{array}{l}\text { Falta de } \\
\text { habilidad } \\
\text { interpersonal }\end{array}$ & 3.6 \\
\hline & & & $\begin{array}{l}\text { Falta de } \\
\text { habilidad } \\
\text { normativa }\end{array}$ & 16.6 & $\begin{array}{l}\text { Falta de } \\
\text { habilidad } \\
\text { normativa }\end{array}$ & 1.8 \\
\hline & & & $\begin{array}{l}\text { Falta de } \\
\text { pensamiento } \\
\text { prospectivo }\end{array}$ & 16.6 & $\begin{array}{l}\text { Falta de } \\
\text { pensamiento } \\
\text { prospectivo }\end{array}$ & 0.9 \\
\hline \multirow{18}{*}{$\begin{array}{c}\text { Factores } \\
\text { externos } \\
\text { positivos } \\
\text { (FE+) }\end{array}$} & Normas (NO) & 100 & Norma social & 100 & Norma social & 15.5 \\
\hline & & & Norma coercitiva & 83.3 & Norma coercitiva & 24.5 \\
\hline & & & Norma mimética & 33.3 & Norma mimética & 1.8 \\
\hline & & & Valores & 16.6 & Valores & 1.8 \\
\hline & Gobierno (GO) & 33.3 & & & Gobierno & 2.7 \\
\hline & $\begin{array}{l}\text { Actitud externa } \\
\text { (ACe) }\end{array}$ & 16.6 & & & Actitud externa & 0.9 \\
\hline & $\begin{array}{l}\text { Hábitos externos } \\
\text { (HAe) }\end{array}$ & 16.6 & & & Hábitos externos & 0.9 \\
\hline & Conciencia (CON) & 66.6 & & & Conciencia & 19.1 \\
\hline & $\begin{array}{l}\text { Contexto externo } \\
\text { (CE) }\end{array}$ & 66.6 & Necesidades & 33.3 & Necesidades & 3.6 \\
\hline & & & Recursos & 16.6 & Recursos & 3.6 \\
\hline & & & Cambio climático & 16.6 & Cambio climático & 1.8 \\
\hline & & & Infraestructura & 16.6 & Infraestructura & 1.8 \\
\hline & $\begin{array}{l}\text { Competencias } \\
\text { (CO) }\end{array}$ & 100 & Acción & 83.3 & Acción & 12.7 \\
\hline & & & $\begin{array}{l}\text { Gestión } \\
\text { estratégica }\end{array}$ & 50 & $\begin{array}{l}\text { Gestión } \\
\text { estratégica }\end{array}$ & 2.7 \\
\hline & & & Normativa & 33.3 & Normativa & 1.8 \\
\hline & & & $\begin{array}{l}\text { Diversificación } \\
\text { e interdiciplina- } \\
\text { riedad }\end{array}$ & 33.3 & $\begin{array}{l}\text { Diversificación e } \\
\text { interdisciplina- } \\
\text { riedad }\end{array}$ & 1.8 \\
\hline & & & $\begin{array}{l}\text { Sistema de } \\
\text { pensamiento }\end{array}$ & 33.3 & $\begin{array}{l}\text { Sistema de } \\
\text { pensamiento }\end{array}$ & 1.8 \\
\hline & & & $\begin{array}{l}\text { Pensamiento } \\
\text { prospectivo }\end{array}$ & 16.6 & $\begin{array}{l}\text { Pensamiento } \\
\text { prospectivo }\end{array}$ & 0.9 \\
\hline \multirow{3}{*}{$\begin{array}{c}\text { Factores } \\
\text { externos } \\
\text { negativos } \\
\text { (FE-) }\end{array}$} & Normas (NO) & 100 & Norma coercitiva & 66.6 & Norma coercitiva & 11.3 \\
\hline & & & Valores & 66.6 & Valores & 6 \\
\hline & & & Norma social & 66.6 & Norma social & 4.6 \\
\hline
\end{tabular}




\begin{tabular}{|c|c|c|c|c|c|c|}
\hline Dimens. & Categoría & $\% *$ & Criterio & $\% *$ & $\begin{array}{c}\text { Criterios } \\
\text { ponderados }\end{array}$ & $\% * *$ \\
\hline \multirow{17}{*}{$\begin{array}{c}\text { Factores } \\
\text { externos } \\
\text { negativos } \\
\text { (FE-) }\end{array}$} & $\begin{array}{l}\text { Contexto externo } \\
\text { (CE) }\end{array}$ & 50 & Infraestructura & 33.3 & Infraestructura & 3.3 \\
\hline & & & Recursos & 33.3 & Recursos & 2 \\
\hline & & & Necesidades & 16.6 & Necesidades & 12.6 \\
\hline & & & Hábilitadores & 16.6 & Habilitadores & 4.6 \\
\hline & & & Cambio climático & 16.6 & Cambio climático & 4 \\
\hline & Corrupción (COR) & 33.3 & & & Corrupción & 2.6 \\
\hline & Inseguridad (IN) & 16.6 & & & Inseguridad & 2.6 \\
\hline & $\begin{array}{l}\text { Falta de } \\
\text { conciencia (CON) }\end{array}$ & 66.6 & & & $\begin{array}{l}\text { Falta de } \\
\text { conciencia }\end{array}$ & 11.3 \\
\hline & $\begin{array}{l}\text { Falta de compe- } \\
\text { tencias (CO) }\end{array}$ & 83.3 & Acción & 83.3 & Falta de acción & 7.9 \\
\hline & & & $\begin{array}{l}\text { Gestión } \\
\text { estratégica }\end{array}$ & 50 & $\begin{array}{l}\text { Falta de gestión } \\
\text { estratégica }\end{array}$ & 3.3 \\
\hline & & & Normativa & 33.3 & $\begin{array}{l}\text { Falta de habilidad } \\
\text { normativa }\end{array}$ & 2 \\
\hline & & & $\begin{array}{l}\text { Pensamiento } \\
\text { prospectivo }\end{array}$ & 33.3 & $\begin{array}{l}\text { Falta de } \\
\text { pensamiento } \\
\text { prospectivo }\end{array}$ & 1.3 \\
\hline & Gobierno (GO) & 50 & & & Gobierno & 11.3 \\
\hline & $\begin{array}{l}\text { Actitud negativa } \\
\text { (AC) }\end{array}$ & 100 & Consumista & 66.6 & Consumista & 4.6 \\
\hline & & & Apatía & 33.3 & Apatía & 2.6 \\
\hline & & & Estilo de vida & 33.3 & Estilo de vida & 1.3 \\
\hline & & & Instrumentista & 16.6 & Instrumentista & 0.7 \\
\hline
\end{tabular}

Fuente: Elaboración propia (2019).

*Total de entrevistados que lo mencionaron

${ }^{* *}$ Cantidad de veces repetido (mencionado).

\section{Factores internos}

La literatura analizada hace mención a la existencia de una serie de elementos que inciden en el fenómeno del comportamiento sostenible, la mayoría se basa en aquellos que provienen del nivel individual por parte de las personas que laboran en una organización y los cuales pueden ejercer un efecto positivo o negativo, dependiendo de la dirección y fuerza con la que se presenten, dentro de los que se pueden mencionar: la intención, las competencias, las actitudes, el marco normativo y los valores (Carro, Reyes, Rosano, Garnica \& Pérez, 2017; Gast et al., 2017; Imtiaz et al., 2017; 
Koe et al., 2014, 2015; Lülfs \& Hahn, 2014; Ploum et al., 2018; Roysen, 2018; Tur-Porcar et al., 2018; Vuorio et al., 2017; Zdanyté et al., 2014).

Comparando las aportaciones previas con los hallazgos encontrados en el presente estudio, se encontró la presencia de la mayoría de los elementos anteriores, brindando así un soporte a los mismos. Los principales factores que influyen de manera interna en la universidad se relacionan con el marco normativo (100\%), específicamente, con los valores (83\%), con la presencia (gestión estratégica $83.3 \%$, y acción $83.3 \%$ ) o ausencia (falta de acción 100\%) de competencias, con la presencia de una conciencia sostenible (100\%) y con la ausencia de recursos (económicos 83.3\%) suficientes para la correcta operación. A partir de lo anterior se afirma que los elementos mencionados están relacionados principalmente con un nivel individual, institucional y contextual.

\section{Factores externos}

En la revisión de la literatura se encontró poca mención sobre los factores externos. Estos factores pueden ejercer una influencia directa o indirecta (positiva o negativa) con relación al actuar de la empresa; no obstante, algunos elementos señalados se relacionan primordialmente con el aspecto situacional del contexto (Lülfs \& Hahn, 2014) y con las normas sociales (Gast et al., 2017; Koe et al., 2014, 2015; Lülfs \& Hahn, 2014; Roysen, 2018).

Los hallazgos encontrados a través del análisis de las entrevistas fundamentan la presencia de algunos elementos externos a la universidad, que influyen en la generación de un comportamiento sostenible. Se encuentra un marco normativo externo favorable (norma social 100\%) o desfavorable (norma coercitiva, valores, norma social, $66.6 \%$ cada una); la presencia (acción 83.3\%) o ausencia (acción 83.3\%) de competencias desde o hacia el exterior y las actitudes negativas, en general (consumismo 66.6\%). Así, entonces, los elementos mencionados están relacionados, principalmente, a un nivel individual, contextual y gubernamental, por lo que se realza la importancia de ciertos elementos posicionados en el exterior que pueden influir directamente en el comportamiento de una organización.

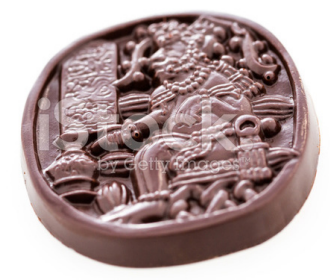




\section{Universidades públicas y privadas}

Sobre las diferencias y similitudes con relación a los elementos que las universidades públicas y privadas consideran más relevantes (tabla 4), se presenta un ranking de los criterios para cada una de las dimensiones de acuerdo con el peso que cada conjunto de unidades aportó a cada criterio. Mientras que para las universidades públicas la presencia de una habilidad de acción (37.8\%) y una adecuada implementación de normas coercitivas (12.2\%) influyen de manera interna y positiva, las universidades privadas ponderan en mayor medida los valores (33.7\%) y la presencia de una conciencia sostenible (25.3\%), estableciéndose así un nivel individual e institucional para las universidades públicas y únicamente un nivel individual para las universidades privadas.

Respecto a los factores negativos internos, las universidades públicas consideran que una actitud consumista (41.5\%) y la falta de acción (14.6\%) limitan la generación de un comportamiento sostenible; mientras que las universidades privadas consideran que, además de la falta de acción (40\%), los recursos económicos limitados (16.7\%) y la falta de una conciencia adecuada (16.7) son los de mayor relevancia. Es así que las universidades públicas se enfocan en elementos a nivel individual, mientras que las universidades privadas lo hacen a un nivel individual e institucional.

En cuanto a los factores externos, las universidades públicas consideran que contar con normas coercitivas favorables desde afuera (37.7\%) y la presencia de una conciencia sostenible por parte de la población (13.1\%) permite fomentar el comportamiento sostenible. Del mismo modo, las universidades privadas también consideran de gran relevancia la presencia de una conciencia (22\%) y adicionan la importancia de una norma o presión social favorable (20.3\%). Ambas universidades ponderan con relevancia los aspectos a nivel contextual para la generación de las conductas mencionadas.

Finalmente, respecto a los elementos externos negativos, las universidades públicas mencionan que el incremento en las necesidades en general (21.6\%) y la falta de una norma coercitiva favorable (13.4\%) son los factores que más limitan las conductas, mientras que las universidades privadas manifiestan que la falta de habilitadores (11.77\%) y la falta de una conciencia (11.7\%) son los principales elementos. Por lo anterior, elementos a nivel contextual y gubernamental son los principalmente mencionados. 
Tabla 4. Ranking de criterios para las universidades públicas y privadas

\begin{tabular}{|c|c|c|c|c|c|c|c|}
\hline \multicolumn{4}{|c|}{ Universidades públicas } & \multicolumn{4}{|c|}{ Universidades privadas } \\
\hline Dimens. & Ranking & Peso del criterio & \%* & Dimens. & Ranking & Peso del criterio & $\% *$ \\
\hline \multirow{14}{*}{ FI+ } & 1 & Acción & 37.8 & $\mathrm{Fl}+$ & 1 & Valores & 33.7 \\
\hline & 2 & Norma coercitiva & 12.2 & & 2 & Conciencia & 25.3 \\
\hline & 3 & Actitud & 8.9 & & 3 & Acción & 13.3 \\
\hline & 4 & $\begin{array}{l}\text { Gestión } \\
\text { estratégica }\end{array}$ & 8.9 & & 4 & $\begin{array}{l}\text { Diversificación e } \\
\text { interdisciplina- } \\
\text { riedad }\end{array}$ & 7.2 \\
\hline & 5 & Valores & 5.6 & & 5 & Norma social & 6 \\
\hline & 6 & Conciencia & 5.6 & & 6 & Edad & 4.8 \\
\hline & 7 & Norma social & 4.4 & & 7 & $\begin{array}{l}\text { Pensamiento } \\
\text { prospectivo }\end{array}$ & 4.8 \\
\hline & 8 & Hábitos & 4.4 & & 8 & Normativa & 2.4 \\
\hline & 9 & $\begin{array}{l}\text { Sistema de } \\
\text { pensamiento }\end{array}$ & 4.4 & & 9 & $\begin{array}{l}\text { Gestión } \\
\text { estratégica }\end{array}$ & 2.4 \\
\hline & 10 & Recursos & 2.2 & & & & \\
\hline & 11 & $\begin{array}{l}\text { Diversificación e } \\
\text { interdisciplina- } \\
\text { riedad }\end{array}$ & 2.2 & & & & \\
\hline & 12 & Normativa & 1.1 & & & & \\
\hline & 13 & $\begin{array}{l}\text { Pensamiento } \\
\text { prospectivo }\end{array}$ & 1.1 & & & & \\
\hline & 14 & Norma mimética & 1.1 & & & & \\
\hline \multirow{11}{*}{ FI- } & 1 & Consumista & 41.5 & $\mathrm{Fl}-$ & 1 & Falta de acción & 40 \\
\hline & 2 & Falta de acción & 14.6 & & 2 & $\begin{array}{l}\text { Recurso } \\
\text { económico }\end{array}$ & 16.7 \\
\hline & 3 & $\begin{array}{l}\text { Recurso } \\
\text { económico }\end{array}$ & 12.2 & & 3 & $\begin{array}{l}\text { Falta de } \\
\text { conciencia }\end{array}$ & 16.7 \\
\hline & 4 & Recurso humano & 9.8 & & 4 & $\begin{array}{l}\text { Falta de habilidad } \\
\text { interpersonal }\end{array}$ & 13.3 \\
\hline & 5 & $\begin{array}{l}\text { Falta de gestión } \\
\text { estratégica }\end{array}$ & 7.3 & & 5 & $\begin{array}{l}\text { Falta de habilidad } \\
\text { normativa }\end{array}$ & 6.7 \\
\hline & 6 & $\begin{array}{l}\text { Falta de } \\
\text { conciencia }\end{array}$ & 7.3 & & 6 & $\begin{array}{l}\text { Falta de gestión } \\
\text { estratégica }\end{array}$ & 3.3 \\
\hline & 7 & $\begin{array}{l}\text { Contexto Interno } \\
\text { inadecuado }\end{array}$ & 2.4 & & 7 & $\begin{array}{l}\text { Falta de } \\
\text { pensamiento } \\
\text { prospectivo }\end{array}$ & 3.3 \\
\hline & 8 & Apatía & 1.2 & & & & \\
\hline & 9 & Resistencia & 1.2 & & & & \\
\hline & 10 & $\begin{array}{l}\text { Hábitos } \\
\text { arraigados }\end{array}$ & 1.2 & & & & \\
\hline & 11 & $\begin{array}{l}\text { Normas } \\
\text { coercitivas } \\
\text { internas } \\
\text { inadecuadas }\end{array}$ & 1.2 & & & & \\
\hline
\end{tabular}




\begin{tabular}{|c|c|c|c|c|c|c|c|}
\hline \multicolumn{4}{|c|}{ Universidades públicas } & \multicolumn{4}{|c|}{ Universidades privadas } \\
\hline \multirow{14}{*}{ FE+ } & 1 & Norma coercitiva & 37.7 & $\mathrm{FE}+$ & 1 & Conciencia & 22 \\
\hline & 2 & Conciencia & 13.1 & & 2 & Norma social & 20.3 \\
\hline & 3 & Acción & 11.5 & & 3 & Necesidades & 13.6 \\
\hline & 4 & Necesidades & 9.8 & & 4 & Acción & 11.9 \\
\hline & 5 & Norma social & 8.2 & & 5 & Norma coercitiva & 6.8 \\
\hline & 6 & Recursos & 6.6 & & 6 & $\begin{array}{l}\text { Diversificación e } \\
\text { interdisciplina- } \\
\text { riedad }\end{array}$ & 3.4 \\
\hline & 7 & Gobierno & 3.3 & & 7 & $\begin{array}{l}\text { Sistema de } \\
\text { pensamiento }\end{array}$ & 3.4 \\
\hline & 8 & Infraestructura & 3.3 & & 8 & Norma mimética & 3.4 \\
\hline & 9 & Actitud externa & 1.6 & & 9 & Valores & 3.4 \\
\hline & 10 & Hábitos externos & 1.6 & & 10 & Cambio climático & 3.4 \\
\hline & 11 & $\begin{array}{l}\text { Gestión } \\
\text { estratégica }\end{array}$ & 1.6 & & 11 & $\begin{array}{l}\text { Gestión } \\
\text { estratégica }\end{array}$ & 3.4 \\
\hline & 12 & Normativa & 1.6 & & 12 & Gobierno & 1.7 \\
\hline & & & & & 13 & Normativa & 1.7 \\
\hline & & & & & 14 & $\begin{array}{l}\text { Pensamiento } \\
\text { prospectivo }\end{array}$ & 1.7 \\
\hline \multirow{17}{*}{ FE- } & 1 & Necesidades & 21.6 & FE- & 1 & Habilitadores & 11.7 \\
\hline & 2 & Norma coercitiva & 13.4 & & 2 & Falta de conciencia & 11.7 \\
\hline & 3 & Gobierno & 11.3 & & 3 & Falta de acción & 10 \\
\hline & 4 & Falta de conciencia & 10.3 & & 4 & Gobierno & 10 \\
\hline & 5 & Falta de acción & 6.2 & & 5 & Valores & 8.3 \\
\hline & 6 & Cambio climático & 6.2 & & 6 & Estilo de vida & 6.7 \\
\hline & 7 & Valores & 4.1 & & 7 & Norma coercitiva & 6.7 \\
\hline & 8 & Norma social & 4.1 & & 8 & Norma social & 5 \\
\hline & 9 & Corrupción & 4.1 & & 9 & $\begin{array}{l}\text { Falta de gestión } \\
\text { estratégica }\end{array}$ & 5 \\
\hline & 10 & Inseguridad & 4.1 & & 10 & $\begin{array}{l}\text { Falta de habilidad } \\
\text { normativa }\end{array}$ & 5 \\
\hline & 11 & Infraestructura & 3.1 & & 11 & Consumista & 5 \\
\hline & 12 & Consumista & 3.1 & & 12 & Apatía & 5 \\
\hline & 13 & Estilo de vida & 3.1 & & 13 & Infraestructura & 3.3 \\
\hline & 14 & $\begin{array}{l}\text { Falta de gestión } \\
\text { estratégica }\end{array}$ & 2.1 & & 14 & Recursos & 3.3 \\
\hline & 15 & Recursos & 1 & & 15 & $\begin{array}{l}\text { Falta de } \\
\text { pensamiento } \\
\text { prospectivo }\end{array}$ & 1.7 \\
\hline & 16 & Apatía & 1 & & 16 & Instrumentista & 1.7 \\
\hline & 17 & $\begin{array}{l}\text { Falta de } \\
\text { pensamiento } \\
\text { prospectivo }\end{array}$ & 1 & & & & \\
\hline
\end{tabular}

${ }^{*}$ Cantidad de veces repetido (mencionado).

Fuente: Elaboración propia (2019). 


\section{Conclusiones}

Los hallazgos y resultados permiten afirmar que la influencia de diversos elementos provenientes tanto del interior como del exterior de las universidades del noreste de Méjico intervienen en el modelamiento de una conducta sostenible. Pese a la existencia de alguna literatura sobre el tema, se hace necesario una mayor exploración el propósito de ampliar los análisis y explicaciones sobre el fenómeno. La investigación abordó un método inductivo que explora e identifica los factores internos y externos respecto a las universidades sostenibles del noreste de Méjico, que afectan positiva y negativamente una conducta sostenible.

En su mayoría, la literatura existente se basa en los aspectos cognitivos del individuo para dar explicación al fenómeno; sin embargo, pocos son los autores y mínima es la profundidad que aborda los aspectos externos a la organización como fuentes de influencia directa, los cuales se hacen presentes en los hallazgos de esta investigación.

Los resultados indican que los impulsores más importantes para un comportamiento sostenible están relacionados con el marco normativo (valores y norma social), las competencias (gestión estratégica, acción) y la presencia de una conciencia favorable, mientras que los aspectos que limitan la generación de la misma son la falta de competencias (acción), la falta de un marco normativo favorable (coercitivo, valores, norma social) y una actitud negativa hacia la sostenibilidad (consumismo). Por lo anterior, elementos a nivel individual, institucional, contextual y gubernamental se hacen presentes.

Los hallazgos permiten, en primera instancia, ampliar la perspectiva del fenómeno del comportamiento sostenible, brindando soporte empírico a las investigaciones previas y abriendo el panorama hacia la influencia de factores más allá de los propios del individuo, es por esto que valdría la pena considerar estos elementos para abordar estudios posteriores. La relevancia de los elementos impulsores de este contexto establece la importancia de su fomento en las organizaciones, especialmente, en el ámbito de las universidades. Como parte de las limitaciones se tiene que la metodología, al ser cualitativa, muestra únicamente las percepciones del entrevistado, por lo que se sugieren estudios posteriores implementando metodologías con técnicas cuantitativas que amplíen los resultados de la presente investigación, tomando en consideración los elementos identificados. 


\section{Referencias bibliográficas}

1. AGUIÑAGA, Eduardo Enrique, HERIQUES, Irene, SCHEEL, Carlos \& SCHEEL, Andrea. (2018). Building resilience: A self-sustainable community approach to the triple bottom line. Journal of Cleaner Production, Vol. 173. Pp.186-196. https://doi. org/10.1016/j.jclepro.2017.01.094

2. AJZEN, Icek. (1991). The Theory of Planned Behavior. Organizational Behavior and Human Decision Processes, Vol. 50, №2. Pp.179-211.

3. AJZEN, Icek. (2002). Perceived Behavioral Control, Self-Efficacy, Locus of Control, and the Theory of Planned Behavior. Journal of Applied Social Psychology, Vol. 34, № 4. Pp.665-683.

4. BANSAL, Pratima \& ROTH, Kendall (2000). Why companies go green: a model of ecological responsiveness. Academy of Management Journal, Vol. 43, No 4. Pp.717736. https://doi.org/10.2307/1556363

5. BAXTER, Pamela \& JACK, Susan. (2008). Qualitative Case Study Methodology: Study Design and Implementation for Novice Researchers. The Qualitative Report, Vol. 13, № 4. Pp. 544-559.

6. BIRD, Barbara. (1988). Implementing Entrepreneurial Ideas: The Case for Intention. Critical Sociolgy, Vol. 13, N³. Pp.442-453. https://doi.org/10.1177/0896920511399938

7. CARRO, Jorge, REYES, Bernardo, ROSANO, Genoveva, GARNICA, Jaime \& PÉREZ, Beatriz. (2017). Modelo de desarrollo sustentable para la industria de recubrimientos cerámicos. Revista Internacional de Contaminación Ambiental, Vol. 33, № 1. Pp.131139. https://doi.org/10.20937/RICA.2017.33.01.12

8. COPE, Jason. (2005). Toward a dynamic learning perspective of entrepreneurship. Entrepreneurship: Theory and Practice, Vol. 29, No 4. Pp.373-397. https://doi. org/10.1111/j.1540-6520.2005.00090.x

9. CRIADO-GOMIS, Ana, CERVERA-TAULET, Amparo \& INIESTA-BONILLO, Maria-Angeles. (2017). Sustainable Entrepreneurial Orientation: A Business Strategic Approach for Sustainable Development. Sustainability, Vol. 9. Pp 1667 https://doi.org/10.3390/ su9091667

10. DIVITO, Lori \& BOHNSACK, René. (2017). Entrepreneurial orientation and its effect on sustainability decision tradeoffs: The case of sustainable fashion firms. Journal of Business Venturing, Vol. 32, № 5. Pp.569-587. https://doi.org/10.1016/j. jbusvent.2017.05.002

11. FERNANDO, Lloyd \& EVANS, Steve. (2016). Competencies to move beyond eco-efficiency. Procedia CIRP, Vol. 40. Pp.365-371. https://doi.org/10.1016/j. procir.2016.01.069

12. GAST, Johanna, GUNDOLF, Katherine \& CESINGER, Beate. (2017). Doing business in a green way: a systematic review of the ecological sustainability entrepreneurship literature and future research directions. Journal of Cleaner Production, Vol.147. Pp.44-56. https://doi.org/10.1016/j.clepro.2017.01.065 
13. HWEE NGA, Joyce Koe \& SHAMUGANATHAN, Gomathi. (2010). The influence of personality traits and demographic factors on social entrepreneurship start up intentions. Journal of Business Ethics, Vol. 95, № 2. Pp.259-282. https://doi. org/10.1007/s10551-009-0358-8

14. IMTIAZ, Aqueel, BLUNDEL, Richard \& SCHAEFER, Anja. (2017). Understanding the drivers of sustainable entrepreneurial practices in Pakistan's leather industry: a multilevel approach. International Journal of Entrepreneurial Behavior \& Research, Vol. 24. №2. Pp. 382-407 https://doi.org/10.1108/JJEBR-11- 2015-0263

15. IPCC, PACHAURI, Rajendra K. \& MEYER, Leo. (2014). Climate Change 2014: Synthesis Report. Intergovernmental Panel on Climate Change, Vol. 1. Pp.1-168.

16. JAHANSHAHI, Asghar Afshar, BREEM, Alexander \& BHATTACHARJEE, Amitab. (2017). Who takes more sustainability-oriented entrepreneurial actions? The role of entrepreneurs' values, beliefs and orientations. Sustainability, Vol. 9, № 10. Pp.1636. https://doi.org/10.3390/su9101636

17. KING, Nigel. (2004). Using interviews in qualitative research. Essential Guide to Qualitative Methods in Organizational Research, Vol. 2. Pp.11-22.

18. KOE, Wei-Loon, OMAR, Roaimah \& MAJID, Izaidin Abdul. (2014). Factors associated with propensity for sustainable entrepreneurship. Procedia-Social and Behavioral Sciences, Vol.130. Pp.65-74. https://doi.org/10.1016/j.sbpro.2014.04.009

19. KOE, Wei-Loon, OMAR, Roaimah \& SA'ARI, Juan Rizal. (2015). Factors influencing propensity to sustainable entrepreneurship of SMEs in Malaysia. ProcediaSocial and Behavioral Sciences, Vol.172. Pp.570-577. https://doi.org/10.1016/j. sbspro.2015.01.404

20. LANS, Thomas, BLOK, Vincent \& WESSELINK, Renate. (2014). Learning apart together: Towards an integrated framework for sustainable entrepreneurship competence in higher education. Journal of Cleaner Production, Vol. 62. Pp. 37-47. http://dx.doi. org/10.1016/j.jclepro.2013.03.036

21. LÜLFS, Rregina \& HAHN, Rüdiger. (2014). Sustainable Behavior in the Business Sphere: a Comprehensive Overviwe of the Explanatory Power of Psychological Models. Organization \& Environment, Vol. 27, No 1. Pp. 43-64. https://doi. org/10.1177/1086026614522631

22. MANESH, Seyed \& RIALP-CRIADO, Alex. (2018). International ecopreneurs: The case of eco-entrepreneurial new ventures in the renewable energy industry. Journal of Inernational Entrepreneurship, Vol.17, No 1. Pp.103-126. https://doi.org/10.1007/ s10843-017-0222-3

23. MARTIN, Roger I. \& OSBERG, Sally. (2007). Social Entrepreneurship: The Case for Definition. Stanford Social Innovation Review, Vol. 15, №2. Pp. 29-39.

24. MILES, Matthew, HUBERMAN, Michael y SALDAÑA, Johnny. (2013). Qualitative Data Analysis: A Methods Sourcebook. $3^{\mathrm{a}}$ ed. California: SAGE. 408 pp. 
25. MUÑOZ, Pablo. (2017). A cognitive map of sustainable decision-making in entrepreneurship: a configurational approach. International Journal of Entrepreneuril Behavior \& Research, Vol. 24, No 3. Pp. 787-813 https://doi.org/10.1108/ IJEBR-03-2017-0110

26. ONU. (2018). Objetivos de Desarrollo Sostenible. Disponible en Internet: http://www. onu.org.mx/agenda-2030/objetivos-del-desarrollo-sostenible/

27. PLOUM, Lisa, BLOK, Vincent, LANS, Thomas \& OMTA, Onno. (2018). Toward a validated competence framework for sustainable entrepreneurship. Organization \& Environment, Vol. 31, № 2. Pp. 113-132. https://doi.org/10.1177/1086026617697039

28. ROYSEN, Rebeca. (2018). O corpo e a adoção de práticas sustentáveis: estudo de caso em uma ecovila. Psicologia \& Sociedade, Vol. 30, № 1. Pp.1-11. https://doi. org/10.1590/1807-0310/2018v30164236

29. RUIZ-RUANO, Ana-María \& PUGA, Jorge. (2016). Sustainable entrepreneurship in universities and environmental values/Emprendimiento sostenible en la universidad y valores ambientales. Psyecology, Vol. 7, № 1. Pp. 1-24. https://doi.org/10.1080/217 11976.2015 .1114218

30. ST-JEAN, Etienne \& LABELLE, Francois. (2018). Wanting to change the world, is it too much of a good thing? How sustainable orientation shapes entrepreneurial behaviour. International Journal of Entrepreneurial Behavior \& Research, Vol. 24, № 6. Pp. 10751086 https://doi.org/10.1108/JJEBR-03-2018-0130

31. SUNG, Chang \& PARK, Joo. (2018). Sustainability Orientation and Entrepreneurship Orientation: Is There a Tradeoff Relationship between Them? Sustainability, Vol. 10, $\mathrm{N}^{\circ}$ 2. Pp.379. https://doi.org/10.3390/su10020379

32. TORRES-HERNÁNDEZ, Tatiana, BARRETO, Idaly \& RINCÓN, Juan Carlos. (2015). Creencias y normas subjetivas como predictores de intención de comprotamiento proambiental. Suma Psicológica, Vol. 22, Pp. 86-92. https://doi.org/10.1016/j.sumpsi.2015.09.003

33. TUR-PORCAR, Ana, ROIG-TIERNO, Norat \& LLORCA MESTRE, Anna. (2018). Factors affecting entrepreneurship and business sustainability. Sustainability, Vol.10. Pp. 452. https://doi.org/10.3390/su10020452

34. URBANIEC, Maria. (2018). Sustainable entrepreneurship: Innovation-related activities in European entreprises. Polish Journal of Environmental Studies, Vol. 27, № 4. Pp. 1773-1779. https://doi.org/10.15244/pjoes/78155

35. VUORIO, Anna Maija, PUUMALAINEN, Kaisu \& FELLNHOFER, Katharina. (2017). Drivers of entrepreneurial intentions in sustainable entrepreneurship. International Journal of Entrepreneurial Behaviour and Research, Vol. 24, № 2. Pp. 359-381. https://doi. org/10.1108/JJEBR-03-2016-0097

36. WAGNER, Marcus \& MAXIMILIANS, Julius. (2012). Ventures for the Public Good and Entrepreneurial Intentions: An Empirical Analysis of Sustainability Orientation as a Determining Factor. Journal of Small Business \& Entrepreneurship, Vol. 25, No 4, Pp. 519-531. https://doi.org/10.1080/08276331.2012.10593587 
37. WESTGREN, Randall \& ZERING, Kelly. (1998). Case Study Research Methods for Firm and Market Research. Agribusiness: An International Journal, Vol. 14, № 5. Pp. 415-423.

38. YIN, Robert. (2003). Case study research: Design and methods. $3^{a}$ ed. California: SAGE. $179 \mathrm{pp}$.

39. ZDANYTÉ, Kristina, NEVERAUSKAS, Bronius \& SABALIAUSKAITÉ, Eglé. (2014). Implementation of sustainable development opportunities in the Lithuanian higher education institution. Procedia-Social and Behavioral Sciences, Vol. 110. Pp. 482-493. https://doi.org/10.1016/j.sbspro.2013.12.892

Para citar este artículo:
RODRÍGUEZ, Lucero de Jesús, SÁNCHEZ, Mónica Lorena y BRISEÑo, Arturo. (2020). Determinantes de un comportamiento sostenible en universidades del noreste de Méjico. Un análisis exploratorio. Teuken Bidikay, Vol. 11, № 16. Medellín: Politécnico Colombiano. Pp. 131-152. doi: 10.33571/teuken.v11n16a6

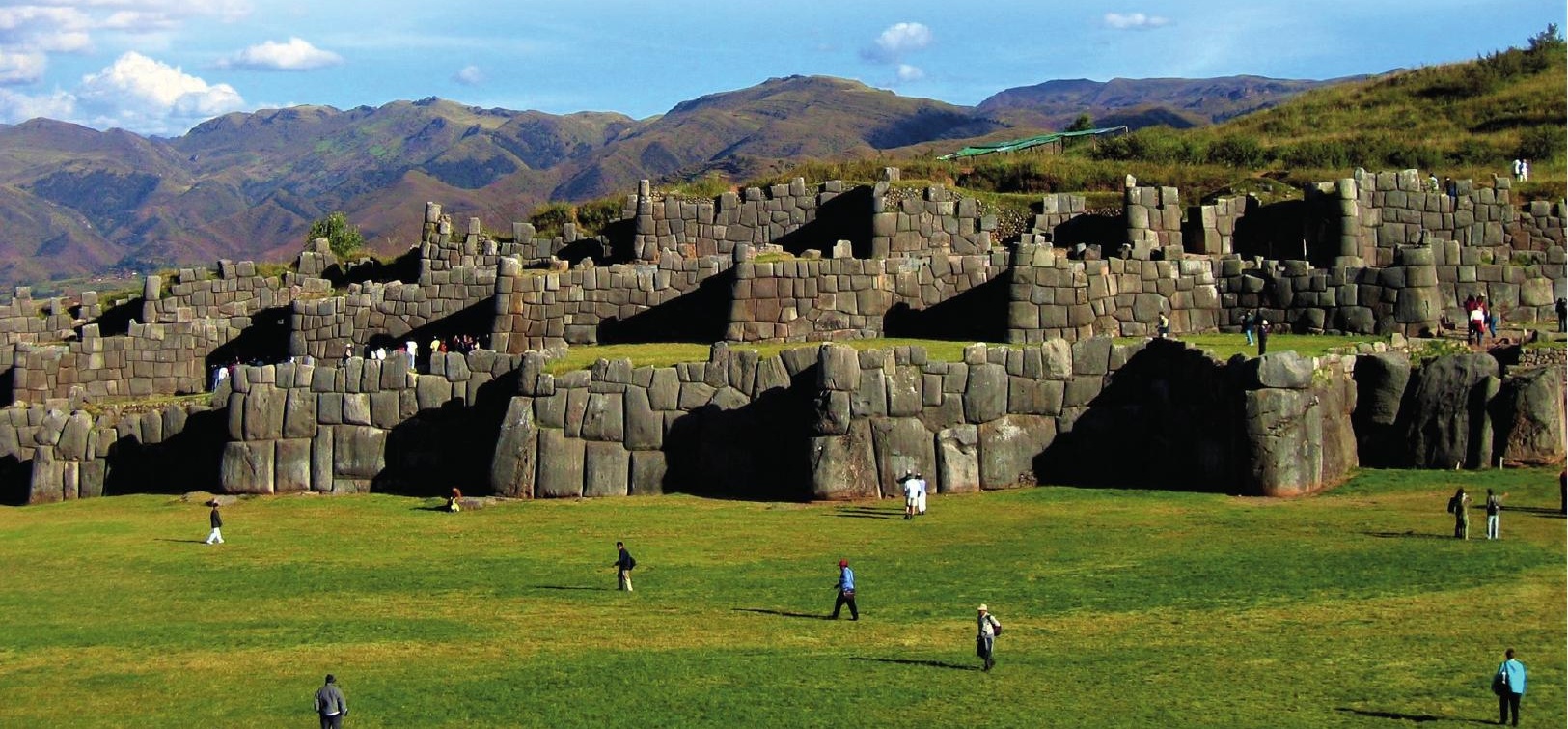

